\title{
A CONTINUITY PROPERTY AND SURFACE TOPOLOGY
}

\author{
BY NEAL R. WAGNER ${ }^{1}$
}

Communicated by R. D. Anderson, April 10, 1973

Introduction. While studying global properties of spaces of retractions of 2-manifolds, the author was led to a continuity property for conformal mappings of simply connected domains with locally connected boundaries $(\S 1$, Theorem 2). Applications are given to the study of spaces of retractions ( 2$)$, spaces of retracts $(\S 3)$, and the Dirichlet problem (§4). Details of the proofs will appear elsewhere.

1. The continuity property. For each nonnegative integer $i$, let $G_{i}$ be a bounded simply connected domain in the plane $E^{2}$ such that each $G_{i}$ contains some fixed closed disk $D$ with the origin as center. We first state a known result.

THEOREM 1 (CARATHÉODORY, ET AL.; BorSUK). For each $i$, the following are equivalent.

(a) The boundary $F_{i}$ of $G_{i}$ is locally connected.

(b) There is a unique continuous surjection $f_{i}$ from the closed unit disk $B^{2}$ to $\operatorname{cl}\left(G_{i}\right)=G_{i} \cup F_{i}$ which fixes the origin, is conformal on $\operatorname{int}\left(B^{2}\right)$, and has positive derivative at the origin.

(c) There is a retraction of $E^{2} \backslash \operatorname{int}(D)$ with image $E^{2} \backslash G_{i}$.

From [8, p. 112] it is clear that $F_{i}$ is locally connected if and only if all the prime ends of $G_{i}$ are of the first kind [4, p. 65], and hence [4, p. 67] (a) implies (b). An elementary construction allows one to prove that (b) implies (c). (Borsuk originally showed directly that (a) implies (c).) It is clear that (b) or (c) implies (a).

By an abuse of language, we consider each point $v \in F_{i}$ as a prime end of the first kind, i.e., as a point with a representing embedding

AMS (MOS) subject classifications (1970). Primary 30A30, 54C15, 54C35, 57A05; Secondary 55D10, 55F05.

Key words and phrases. Absolute neighborhood retract, compact-open topology, conformal mapping, continuity property, cross section, Dirichlet problem, Fréchet convergence, function space, homotopy equivalence, prime end of the first kind, retract, retraction, Riemann surface, 2-manifold.

${ }^{1}$ The author is indebted to Mary-Elizabeth Hamstrom, who served as his thesis advisor during the early stages of this research. He would also like to thank the referee of [6] for some very useful ideas. 
$e:[0,1] \rightarrow G_{i} \cup F_{i}$, where $e([0,1)) \subset G_{i}$ and $e(1)=v$. A sequence $\left\{v_{i} \in F_{i}: i \geqq 1\right\}$ of prime ends converges to a prime end $v_{0} \in F_{0}$ if we can select representing embeddings $e_{i}$ for $v_{i}$ such that $\left\{e_{i}: i \geqq 1\right\}$ converges uniformly to $e_{0}$ on $[0,1]$.

Definition. The sequence $\left\{F_{i}: i \geqq 1\right\}$ Fréchet converges to $F_{0}$ if (1) each prime end $v_{0} \in F_{0}$ is the limit of a sequence $\left\{v_{i} \in F_{i}: i \geqq 1\right\}$ of prime ends, and (2) each subsequence of a sequence $\left\{v_{i} \in F_{i}: i \geqq 1\right\}$ of prime ends has, in turn, a subsequence which converges to a prime end of $F_{0}$.

See [1] for other generalizations of Fréchet convergence (also termed 0 -regular convergence [3], [7]) and for a result similar to (and more general than) the equivalence of (a) and (b) in Theorem 2 below.

THEOREM 2. The following are equivalent.

(a) The sequence $\left\{F_{i}: i \geqq 1\right\}$ Fréchet converges to $F_{0}$.

(b) The sequence $\left\{f_{i}: i \geqq 1\right\}$ converges uniformly to $f_{0}$ on the closed disk $B^{2}$.

(c) There are retractions $\phi_{i}$ of $E^{2} \backslash \operatorname{int}(D)$ with image $E^{2} \backslash G_{i}$ such that the sequence $\left\{\phi_{i}: i \geqq 1\right\}$ converges uniformly to $\phi_{0}$ on $E^{2} \backslash \operatorname{int}(D)$.

The proof that (a) implies (b) can be carried out with Lindelöf's lemma using a modification of the proof in [2, pp. 59-62]. The proof that (b) implies (c) is similar to the corresponding proof in Theorem 1. A fairly complicated proof shows that the denial of (a) implies the denial of (c).

Using covering spaces, Theorems 1 and 2 generalize to precompact simply connected domains with locally connected boundaries on an arbitrary 2-manifold.

2. The space of retractions. We shall consider two components of the space of retractions of any compact 2-manifold with the compact-open ( = sup-metric) topology.

THEOREM 3. The space of deformation retractions of $M^{2}$ is contractible in itself.

The contraction can be constructed directly using the conformal mappings of Theorem 2(b).

Let $\Lambda$ be the embedding of $M^{2}$ into the space of nullhomotopic retractions of $M^{2}$ (= retractions with contractible image) which maps each point to the constant retraction to that point.

THEOREM 4. The embedding $\Lambda$ is a homotopy equivalence with homotopy inverse the map ev which evaluates a retraction at a basepoint of $M^{2}$.

The proof is similar to the proofs in [6] and [7], except that here we use a cross section theorem [5, p. 55] instead of Michael's selection theorem. 
We also use the fact that the space of simple closed curves in $M^{2}$ is an ANR [3].

3. The space of retracts. The set of retracts (= images of retractions) of any compact 2-manifold $M^{2}$ is given a natural quotient topology of the space of retractions of $M^{2}$ by saying that a sequence of retracts $\left\{R_{i}: i \geqq 1\right\}$ converges to $R_{0}$ if there are retractions $\phi_{i}$ of $M^{2}$ with image $R_{i}(i \geqq 0)$ such that $\left\{\phi_{i}: i \geqq 1\right\}$ converges uniformly to $\phi_{0}$ on $M^{2}$.

THEOREM 5. (i) The space of retracts of $M^{2}$ is an $A N R$.

(ii) The space of deformation retracts of $M^{2}$ is an $A R$.

(iii) The space of compact AR subsets of $M^{2}$ is an ANR with a homeomorphic copy of $M^{2}$ as a deformation retract.

We use the fact that any retract of $M^{2}$ will be a deformation retract of a submanifold $N^{2}$ of $M^{2}$, where $N^{2}$ is a retract of $M^{2}$. The space of deformation retracts of $N^{2}$ is homeomorphic to a retract of a product of ANR's (one for each hole of $N^{2}$ ), where the ANR's are the space of those maps of $B^{2}$ into a 2-manifold $L^{2}$ (obtained by filling in the holes of $N^{2}$ ) which send the origin to a fixed $u_{0} \in L^{2}$, are conformal on int $\left(B^{2}\right)$, and send the positive $x$-axis to a fixed direction at $u_{0}$. The latter space is an $r$-image of a retract of a space studied in [3, Lemma 9].

4. The Dirichlet problem. In this section we assume $M^{2}$ is an orientable Riemann surface whose interior has a continuous nonvanishing vector field. Let $\mathscr{F}$ be any collection of locally connected continua in $M^{2}$ such that each $F \in \mathscr{F}$ is the boundary of a simply connected domain $G_{F}$, and give $\mathscr{F}$ the Fréchet topology. We can use the cross-section theorem of [5, p. 55] to produce continuously, for each $F \in \mathscr{F}$, a point $u_{F} \in G_{F}$, and we then obtain a canonical continuous function $f_{F}: B^{2} \rightarrow G_{F} \cup F$ which is conformal on $\operatorname{int}\left(B^{2}\right)$, takes the origin to $u_{F}$, and takes the direction of the positive $x$-axis to the direction given by the vector field.

For each $F \in \mathscr{F}$, suppose there is given a continuous real-valued function $b_{F}$ defined on $F$ such that the function $B$ defined on $\mathscr{F}$ by $B(F)=b_{F} \circ\left(\left.f_{F}\right|_{S^{1}}\right)$ is continuous, where the range is the space of continuous real-valued functions on $S^{1}$ with the sup-metric topology. (For example, one could use any continuous real-valued function $b$ defined on the union of the members of $\mathscr{F}$ and set $b_{F}=\left.b\right|_{F}$.) Let $\mathscr{G} \subset \mathscr{F} \times M^{2}$ consist of all $(F, u)$ such that $u \in G_{F} \cup F$.

THEOREM 6. There is a continuous real-valued function $h$ defined on $\mathscr{G}$ such that for fixed $F \in \mathscr{F}, h(F, \cdot)$ is harmonic on $G_{F}$ and agrees with $b_{F}$ on $F$.

\section{REFERENCES}

1. D. Gaier, Über die konforme Abbildung veränderlicher Gebiete, Math. Z. 64 (1956), 385-424. MR 17, 1191. 
2. G. M. Goluzin, Geometric theory of functions of a complex variable, GITTL, Moscow, 1952; English transl., Transl. Math. Monographs, vol. 26, Amer. Math. Soc., Providence, R.I., 1969. MR 15, 112; MR 40 \# 308.

3. R. Luke and W. K. Mason, The space of homeomorphisms on a compact 2-manifold is an absolute neighborhood retract, Trans. Amer. Math. Soc. 164 (1972), 275-285.

4. A. I. Markuševič, Theory of functions of a complex variable. Vol. III, GITTL, Moscow, 1950; English transl., Prentice-Hall, Englewood Cliffs, N.J., 1967. MR 12, 87; MR 35 \#6799.

5. N. E. Steenrod, The topology of fibre bundles, Princeton Math. Series, vol. 14, Princeton Univ. Press, Princeton, N.J., 1951. MR 12, 522.

6. N. R. Wagner, The space of retractions of the 2-sphere and the annulus, Trans. Amer. Math Soc. 158 (1971), 319-329. MR 43 \#5484.

7. - The space of retractions of a 2-manifold, Proc. Amer. Math. Soc. 34 (1972), 609-614. MR 45 \#4350.

8. G. T. Whyburn, Analytic topology, Amer. Math. Soc. Colloq. Publ., vol. 28, Amer. Math. Soc., Providence, R.I., 1942. MR 4, 86.

Department of Mathematics, University of TeXas, El Paso, Texas 79968 